\title{
Brine Shrimp Toxicity of Essential Oils from Musa spp.
}

\author{
Mohd Alhafiizh Zailani*1 \& Abdul Razzak Fikri Sharkawi ${ }^{2}$ \\ ${ }^{1}$ Centre for Pre-University Studies, Universiti Malaysia Sarawak, 94300 Kota Samarahan, Sarawak, \\ Malaysia; ${ }^{2}$ Faculty of Resource Science and Technology, Universiti Malaysia Sarawak, 94300 Kota \\ Samarahan, Sarawak, Malaysia \\ *Corresponding author: zmalhafiizh@unimas.my \\ Received: 17 January 2019 Accepted: 19 March $2019 \quad$ Published: 30 June 2019
}

\begin{abstract}
Essential oils of seven Musa spp.'s fruits namely, Musa acuminata colla 'gros michel' (PE), Musa acuminata colla 'lakatan' (PB), Musa acuminata colla 'sucrier' (PM), Musa acuminata $\times$ balbisiana 'horn plantain' (PT), Musa acuminata $\times$ balbisiana colla 'saba' (PN), Musa acuminata colla 'inarnibal' (PO) and Musa acuminata colla 'red' (PJ) were extracted by hydrodistillation method using Clevenger apparatus. The essential oils were analysed using GC-FID and identified using Kovat Indeces compared with published information. PE gave the highest yields of oils compared to the other species with yield of $0.43 \%$ and $0.28 \%$ for its peels and flesh, respectively. The most abundant groups present in most of the essential oils are ester and alcohol groups. The brine shrimp toxicity of PT, PM, PN and PO essential oils were tested and showed toxicity against brine shrimp in dose dependent manner. It can be concluded that the essential oils showed some biological activities which may be a potent medicine in curing tumor. This study implies that the presence of ester and alcohol groups are a good marker for the biological activities of plants. Further studies should focus on the potential of the essential oils of Musa spp. as an antitumor medicine.
\end{abstract}

Keywords: Artemia salina, banana, essential oil, hydrodistillation

Copyright: This is an open access article distributed under the terms of the CC-BY-NC-SA (Creative Commons Attribution-NonCommercial-ShareAlike 4.0 International License) which permits unrestricted use, distribution, and reproduction in any medium, for non-commercial purposes, provided the original work of the author(s) is properly cited.

\section{INTRODUCTION}

Essential oils are very valuable natural product that are used as raw materials in many fields such as food preservation, pharmaceuticals, alternative medicine, perfumes, cosmetics, aromatherapy, spiritual uses, spices and nutrition (Buchbauer, 2000).

The presence of chemical constituent in the essential oil are very valuable due to their biological activities such as antibacterial (Dutra et al., 2019), antifungal (Sienkiewiez et al., 2015), anti-inflammatory (Murbach et al., 2014; Koh et al., 2002), anti-lice (Di et al., 2012), anti-tumour (Calcabrini et al., 2004), antioxidant (Aazza et al., 2014) and act as insect repellent (Gkinis et al., 2003).

Musa spp. commonly known as banana, is a tropical plant that has been consumed since many years ago by mankind and animals for its nutrients and delicious taste. In Malaysia, Musa spp. is known as pisang. It is one of local favorite fruits, which is used for cooking and making desserts.

Musa spp. comes from Musaceae family and approximately 1200 species of varieties are reported (Guylène et al., 2008). Analytical research on the volatile compound of Musa spp. had been carried out for more than 55 years with various extraction methods (Shiota, 1993; Brat et al., 2004; Aurore et al., 2011). Generally, volatile compound of Musa spp. consists of three major components, which are ester, alcohol, and carbonyl groups (ketones and aldehydes) (Jordán et al., 2001; Pérez et al., 1997). This chemical constituent that is present in Musa spp. is valuable, especially in traditional and alternative medicine in countries such as India, Pakistan, United States and Asian countries such as Malaysia, Thailand, Philippines and Indonesia because of the biological activities of the fruit (Pari \& Umamaheswari, 2000; O'Hara et al., 1998; Orhan, 2001). Thus, the objective of this study was to evaluate the brine shrimp toxicity and antibacterial properties of this plants' essential oils which were extracted using hydro-distillation technique. 


\section{MATERIALS AND METHODS}

\section{Sample Preparation}

Seven Musa spp. fruits namely M. acuminata colla 'gros michel' (PE), M. acuminata colla 'lakatan' (PB), M. acuminata colla 'sucrier' (PM), M. acuminata $\times$ balbisiana 'horn plantain' $(\mathrm{PT}), M$. acuminata $\times$ balbisiana colla 'saba' $(\mathrm{PN}), M$. acuminata colla 'inarnibal' (PO) and M. acuminata colla 'red' (PJ) were purchased from local markets in Kuching and Kota Samarahan. The fresh ripe samples were separated into peels and flesh. Each part of the samples was sliced to small pieces. About $100 \mathrm{~g}$ to $500 \mathrm{~g}$ of the sample was weighed using a single pan electronic weighing balance and placed into a 3 L flat-round bottom flask.

\section{Extraction of Essential Oils}

The essential oil of seven Musa spp.'s fruits were extracted using hydrodistillation method in a Clevenger-type apparatus based on method suggested by Sim et al. (2013). Fresh samples, which were placed into a $3 \mathrm{~L}$ flat-round bottom flask were added together with $1.5 \mathrm{~L}$ of distilled water. The sample was hydro-distillated continuously for 6 hours. The essential oils were diluted in $n$-hexane upon the collection and left to cool at room temperature. The oils were separated from water by passing them over anhydrous sodium sulphate. The essential oils were kept in 2-mL amber glass vial and kept in a refrigerator at $4{ }^{\circ} \mathrm{C}$ before further analysis. The percentage yield of essential oils was calculated based on the weight of the essential oils extracted over dry weight of the sample after the extraction.

\section{Gas Chromatography - Flame Ionisation Detector (GC-FID) Analysis}

The constituents of the essential oils were analysed using Gas Chromatography-Flame Ionisation Detector (GC-FID). Exactly, $1 \mu \mathrm{L}$ of sample was diluted with $199 \mu \mathrm{L} n$-hexane prior to the analysis. The samples were analysed in gas chromatography (GC) model of Agilent Hewlett-Packard (HP) 6890 equipped with flame ionisation detector (FID) using capillary HP-5 column $(30 \mathrm{~m} \times 250 \mu \mathrm{m} \times \quad 0.25$ $\mu \mathrm{m})$. The carrier gas used was purified helium. The initial temperature for the oven was set at $50^{\circ} \mathrm{C}$ for 5 minutes and increased to $280{ }^{\circ} \mathrm{C}$ with the rate of 6.5 ${ }^{\circ} \mathrm{C} \min ^{-1}$ and hold for 7 minutes.
Exactly, $1 \mu \mathrm{L}$ of diluted sample was injected in the GC-FID by splitless injection mode. Standard of $n$-alkanes with internal standard of $\mathrm{C}_{20} \mathrm{H}_{42}$ was also analysed as a standard for the calculation of Kovat's Indeces. The individual components present in the essential oils were determined based on their calculated Kovat's Index by comparison with previous published data.

\section{Kovat's Index}

Kovat's Index was used to identify the chemical constituents that are present in the essential oils. The Kovat's Index was calculated using Eq. 1.

$I_{x}=100\left[\frac{t_{x}-t_{n}}{t_{(n+1)}-t_{n}}\right]+100 n \quad$ Eq. 1

Where,

$\mathrm{I}_{x}=$ Kovat's Index

$\mathrm{t}_{x}=$ Retention time for compound $\mathrm{x}$

$t_{n}=$ Retention time of alkane eluted before the compound

$t_{(n+1)}=$ Retention time of alkane eluted after the compound

$\mathrm{n}=$ Number of carbon atom

\section{Semi-Qualitative Analysis}

The percentage of individual chemical components in the essential oil were calculated using a normalised calculation as in Eq. 2.

$\mathbf{\%} \mathbf{X}=\frac{\mathbf{A}_{\mathbf{X}}}{\Sigma \mathbf{A}_{\mathbf{1}}} \times \mathbf{1 0 0 \%} \quad$ Eq. 2

Where,

$\mathrm{A}_{x}=$ Peak area of $\mathrm{X}$

$\Sigma \mathrm{A}_{1}=$ Sum of all area (that identified in the compound of essential oil only)

\section{Percentage Yield}

The percentage yield of essential oils was calculated based on the dry weight of sample used for each extraction. The percentage yields were calculated using Eq. 3. 
Percentage yield $(\%)=$

$\frac{\text { Weight of oil }(\mathrm{g})}{\text { Weight of the dried sample }(\mathrm{g})} \times 100 \% \quad$ Eq. 3

\section{Cluster Analysis}

Cluster analysis was performed using SPSS version 22. Cluster analysis was used to identify whether the species are related to each other. From this information, the data can be used for the chemotaxonomy classification.

\section{Brine Shrimp Toxicity Test}

Cytotoxicity test of essential oils of Musa spp. was tested against brine shrimp, Artemia salina using method described by McLaughlin and Rogers (1998). The brine shrimp eggs were hatched in a beaker containing artificial sea water which was prepared using $38 \mathrm{~g}$ of artificial sea salt in $1 \mathrm{~L}$ of distilled water and left for 2 days at room temperature. Stock solutions of samples with a concentration of $1000 \mathrm{ppm}$ were prepared. Exactly $2 \mu \mathrm{L}, 20 \mu \mathrm{L}$ and $200 \mu \mathrm{L}$ of stock solution transferred were into a separate hole of 24 well NUNC multiwell and left to dry the solvent. Methanol was used as negative control as it was added in the multiwell and were left to dry. Exactly $5 \mu \mathrm{L}$ of dimethylsulphoxide (DMSO) and $2 \mathrm{~mL}$ of artificial seawater was transferred into each hole including the control holes. Exactly 10 Artemia salina larvae were added into each hole of the multiwell plates. The multiwell plates were left for one day under room temperature with exposure to light. The number of dead shrimp was counted and percentage of mortality was determined.

\section{Antibacterial Assay}

The antibacterial activity of essential oils of Musa spp. was determined by disc diffusion based on method by Sim et al. (2013). The bacteria used were Staphylococcus aureus, Staphylococcus epidermidis and Pseudomonas aeruginosa. The pure cultures of all bacteria were inoculated into Mueller-Hinton broth and incubated at $37^{\circ} \mathrm{C}$ for 24 hours. The optical density (OD) of each bacterium was standardised to $0.168 \mathrm{OD}$ at $610 \mathrm{~nm}$ wavelength using UV-Vis spectrophotometer. Commercial penicillin $\left(10 \mu \mathrm{g} \mathrm{disc}{ }^{-1}\right)$ and streptomycin $\left(10 \mu \mathrm{g} \mathrm{disc}{ }^{-1}\right)$ were used as positive controls. Sterile discs loaded with methanol were used as the negative control. The agar plates were prepared using Mueller-Hinton agar. The agar plates were swabbed with bacterial culture uniformly using sterile cotton uniformly. Stock solutions of samples were prepared at a concentration of $1000 \mathrm{ppm}$. Then $100 \mu \mathrm{L}$ of stock solution was loaded on $6 \mathrm{~mm}$ sterile discs. The discs were placed on the surface of medium and the agar plates were incubated at $37{ }^{\circ} \mathrm{C}$ for 24 hours. The inhibition zones were measured in millimeter. The assay was conducted in triplicates.

\section{RESULTS AND DISCUSSION}

\section{Essential Oil Yields}

The percentage yield of essential oils obtained from seven Musa spp. are varied. The percentage yield, the physical appearance of the extracted essential oils are shown in Table 1.

The percentage yield of essential oils extracted from the flesh part of the fruits are in a range of $0.06 \%(\mathrm{w} / \mathrm{w})$ to $0.28 \%(\mathrm{w} / \mathrm{w})$. The highest percentage yield among the flesh samples was shown by $M$. acuminata colla 'gros michel' with a yield of $0.28 \%(\mathrm{w} / \mathrm{w})$, while the lowest yield was obtained from $M$. acuminata colla 'red' with a yield of $0.06 \%$ $(w / w)$. The essential oils extracted from the peels part gave yields in a range of $0.05 \%(\mathrm{w} / \mathrm{w})$ to $0.43 \%$ (w/w). M. acuminata colla 'gros michel' peels have the highest yield with a yield of $0.43 \%(\mathrm{w} / \mathrm{w})$, while the lowest yield was given by $M$. acuminata $\times$ balbisiana colla 'saba' with a percentage of $0.05 \%(\mathrm{w} / \mathrm{w})$.

\section{Chemical Composition of Essential Oils}

All essential oils of Musa spp. were extracted by hydrodistillation method showed different chemical composition between the studied species. The chemical compositions of essential oils from seven Musa spp. is shown in Table 2.

The chemical constituents in essential oil of Musa spp. consist of a complex mixture mainly esters, alcohols, carboxylic acids, aldehydes, ketones, and terpenes with a total of 247 components were identified. The essential oils of Musa spp. studied showed most of the components are from the alcohol, ester and carbonyl group. This is similar to previous studies by Selli et al. (2012), Nogueira et al. (2003) and Jordán et al. (2001), where these three groups were the most abundant groups in Musa spp. essential oils. Each class of chemical components in Musa spp.'s fruit are important in determining the properties and aromatic quality of the Musa spp. (Aurore et al., 2011). 
Table 1. The percentage yield and the physical appearance of the extracted essential oils

\begin{tabular}{|c|c|c|c|c|c|}
\hline Species & Part & $\begin{array}{l}\text { Percentage yield } \\
\text { of oil }(\%)\end{array}$ & Colour & Major Component & $\begin{array}{c}\text { Percentage } \\
\text { Composition (\%) }\end{array}$ \\
\hline \multirow{2}{*}{ PM } & Peels & 0.32 & Light yellow & $\alpha$-Terpinyl acetate & 7.72 \\
\hline & Flesh & 0.23 & Light yellow & $\beta$-Phellandrene & 9.56 \\
\hline \multirow{2}{*}{ PN } & Peels & 0.05 & Colourless & Eugenol acetate & 19.75 \\
\hline & Flesh & 0.08 & Light yellow & $\alpha$-Cadinene & 49.39 \\
\hline \multirow{2}{*}{ PT } & Peels & 0.09 & Light yellow & Ethyl decanoate & 36.63 \\
\hline & Flesh & 0.12 & Light yellow & 13-Epi-manool & 18.77 \\
\hline \multirow{2}{*}{ PE } & Peels & 0.43 & Colourless & Piperitenone & 8.52 \\
\hline & Flesh & 0.28 & Colourless & Ethyl pentanoate & 33.80 \\
\hline \multirow{2}{*}{$\mathrm{PO}$} & Peels & 0.32 & Light yellow & Epi-1-cubenol & 22.77 \\
\hline & Flesh & 0.13 & Colourless & Isospathulenol & 5.26 \\
\hline \multirow{2}{*}{ PJ } & Peels & 0.09 & Colourless & Dehydro-1,8-cineole & 34.47 \\
\hline & Flesh & 0.06 & Colourless & Palustrol & 20.20 \\
\hline \multirow{2}{*}{ PB } & Peels & 0.22 & Pale yellow & Ethyl tetradecanoate & 16.26 \\
\hline & Flesh & 0.12 & Pale yellow & Benzyl benzoate & 12.48 \\
\hline
\end{tabular}

Notes : PM - M. acuminata colla 'sucrier', PN - M. acuminata $\times$ balbisiana colla 'saba', PT - M. acuminata $\times$ balbisiana 'horn plantain', PE - M. acuminata colla 'gros michel', PO - M. acuminata colla 'inarnibal', PJ - M. acuminata colla 'red', PB - M. acuminata colla 'lakatan'

Table 2. Chemical constituents of seven Musa spp.'s fruit essential oils

\begin{tabular}{|c|c|c|c|c|c|c|c|c|c|c|c|c|c|c|}
\hline \multirow{2}{*}{ Compound } & \multicolumn{2}{|c|}{ PM } & \multicolumn{2}{|c|}{ PN } & \multicolumn{2}{|c|}{ PO } & \multicolumn{2}{|c|}{ PT } & \multicolumn{2}{|c|}{ PJ } & \multicolumn{2}{|c|}{ PE } & \multicolumn{2}{|c|}{ PB } \\
\hline & $\mathbf{F}$ & $\mathbf{S}$ & $\mathbf{F}$ & $\mathbf{S}$ & $\mathbf{F}$ & $\mathbf{S}$ & $\mathbf{F}$ & $\mathbf{S}$ & $\mathbf{F}$ & $\mathbf{S}$ & $\mathbf{F}$ & $\mathbf{S}$ & $\mathbf{F}$ & $\mathbf{S}$ \\
\hline \multicolumn{15}{|c|}{ Alcohol } \\
\hline$(2 E, 6 E)$-Farnesol & 0.15 & 0.44 & 0.40 & 0.30 & - & 0.18 & - & - & 2.36 & 0.27 & 0.39 & 0.78 & 1.01 & 5.28 \\
\hline (2Z,6Z)-Farnesol & 0.30 & 0.24 & - & - & - & - & - & - & - & - & - & - & - & - \\
\hline (E)-2-Hexen-1-ol, acetate & - & - & - & - & 0.27 & - & - & - & - & - & - & - & - & - \\
\hline (E)-2-Octen-1-ol & - & - & - & - & - & - & - & - & - & 0.07 & - & - & - & - \\
\hline (E)-Nerolidol & - & - & - & - & - & - & - & - & 0.19 & - & - & - & - & - \\
\hline (Z)-Nerolidol & - & - & 0.54 & 0.48 & 0.07 & - & - & 0.10 & 0.94 & - & - & - & - & 0.53 \\
\hline 1,10-di-epi-Cubenol & - & 0.63 & - & - & - & - & - & - & - & - & - & - & - & - \\
\hline 10-epi- $\gamma$-Eudesmol & 0.88 & 0.26 & - & - & - & - & - & 0.12 & 0.04 & - & 0.13 & 0.26 & 0.57 & - \\
\hline 13-epi-Manool & 0.05 & - & - & - & - & - & 18.77 & - & 0.07 & 0.31 & - & 0.39 & - & - \\
\hline 1-Heptanol & - & - & - & - & - & - & - & - & 0.06 & 0.34 & 0.17 & - & - & - \\
\hline 1-Hexadecanol & 2.64 & - & - & - & 0.11 & - & 0.25 & - & - & 0.31 & 0.09 & 0.31 & 0.11 & 0.20 \\
\hline 1-Octadecanol & 0.59 & - & - & - & 0.27 & - & - & - & - & - & - & - & 0.21 & - \\
\hline 1-Octanol & 0.10 & - & - & - & - & - & - & - & 0.07 & - & - & - & - & - \\
\hline 1-Tetradecanol & 0.90 & 0.12 & 2.00 & 5.22 & - & 2.54 & - & - & - & - & 0.34 & 0.80 & 0.44 & - \\
\hline 2-methoxy-p-Cresol & - & - & - & - & - & - & - & - & - & - & - & 0.12 & - & - \\
\hline 3-Octanol & 0.13 & - & - & 8.24 & - & - & - & - & - & - & - & - & - & - \\
\hline 6-methyl-5-Hepten-2-ol & - & - & - & - & - & 6.46 & - & - & - & - & 27.33 & - & - & - \\
\hline Carotol & - & - & - & - & - & - & - & 0.28 & - & - & - & - & 0.36 & - \\
\hline Carvacrol & - & - & - & - & - & - & - & - & - & - & - & - & - & - \\
\hline Caryophylla-4(12),8(13)-dien-5 $\alpha$-ol & 0.16 & - & 0.39 & - & 5.88 & - & - & - & - & - & - & 0.17 & - & - \\
\hline cis-1(7),8-p-Menthadien-2-ol & - & - & - & - & 0.05 & - & - & - & - & - & - & - & - & - \\
\hline cis-Carveol & - & - & - & - & 0.07 & - & - & - & 0.43 & - & - & - & 0.45 & - \\
\hline cis-Piperitol & - & - & - & - & 0.09 & - & - & - & 0.07 & - & - & - & - & - \\
\hline Citronellol & 0.03 & - & - & - & - & - & - & - & - & - & - & - & - & - \\
\hline Cubebol & - & - & - & 13.13 & 3.49 & - & - & 0.26 & 2.92 & - & 0.51 & - & - & - \\
\hline Elemol & - & 0.42 & 0.29 & 1.98 & - & - & - & - & - & - & - & 0.06 & 1.55 & - \\
\hline epi-1-Cubenol & - & - & 0.24 & 0.48 & 5.51 & 22.77 & - & - & 0.04 & - & - & 0.02 & 0.22 & 0.07 \\
\hline epi-Globulol & - & 0.35 & - & - & - & - & - & - & - & - & - & - & - & - \\
\hline epi- $\alpha$-Bisabolol & 0.17 & 0.40 & 0.71 & 0.46 & - & - & 0.30 & - & 0.13 & 0.14 & - & - & - & - \\
\hline epi- $\alpha$-Cadinol & 0.04 & 0.15 & - & - & - & - & - & 1.74 & - & - & - & - & - & - \\
\hline Eudesma-7(11)-en-4-ol & - & - & 0.42 & 0.91 & - & 0.42 & 0.19 & - & 1.41 & - & - & 0.09 & - & - \\
\hline Eudesma-4(15),7-dien-1 $\beta$-ol & 0.30 & - & - & - & - & - & - & 0.11 & - & - & - & - & 0.17 & 0.09 \\
\hline Eugenol & 8.83 & - & - & - & - & - & - & - & - & - & - & 0.03 & - & - \\
\hline Germacrene- $\delta$-4-ol & - & 3.26 & - & 0.58 & - & - & - & - & - & - & - & - & 1.57 & - \\
\hline Guaiol & 0.95 & 6.10 & - & - & - & - & - & - & - & - & - & - & - & - \\
\hline Himachalol & - & - & - & - & 1.11 & 0.09 & - & 0.20 & - & - & 0.17 & 0.31 & 1.18 & 0.34 \\
\hline Intermedeol & - & - & 0.94 & 1.12 & 0.65 & 11.64 & - & 0.20 & 2.50 & - & 0.13 & - & 0.45 & - \\
\hline Isoborneol & - & - & - & - & - & - & - & - & - & - & - & 4.54 & - & - \\
\hline iso-Dihydrocarveol & - & - & - & - & 0.23 & - & - & - & - & - & - & 0.02 & - & - \\
\hline Isophytol & - & - & - & - & - & - & - & - & 0.12 & - & - & - & - & - \\
\hline Isophytol & - & 0.56 & - & - & - & - & 0.99 & 1.11 & - & 0.37 & - & 0.38 & 0.66 & 0.07 \\
\hline Isospathulenol & 0.93 & 0.53 & - & - & 8.86 & - & - & 0.17 & - & - & - & 0.33 & 0.08 & 0.45 \\
\hline Linalool & - & - & - & - & - & - & - & - & - & - & - & 0.40 & - & - \\
\hline Manool & - & - & - & - & - & - & - & 0.12 & - & - & - & 0.33 & - & - \\
\hline Methyl ether thymol & - & - & - & - & - & - & - & - & - & - & - & 0.02 & - & - \\
\hline
\end{tabular}




\begin{tabular}{|c|c|c|c|c|c|c|c|c|c|c|c|c|c|c|}
\hline \multicolumn{15}{|l|}{ Continue from Table 2} \\
\hline Methyl eugenol & - & - & - & - & - & - & - & - & - & - & - & 0.07 & - & - \\
\hline Palustrol & 3.15 & - & - & - & - & - & - & - & 20.20 & - & - & - & - & - \\
\hline Patchouli alcohol & 0.43 & 0.32 & - & - & 2.54 & - & 0.26 & - & 1.06 & 0.14 & 0.39 & 1.36 & 0.90 & 0.22 \\
\hline$p$-Cresol & - & 0.21 & - & - & - & - & 2.67 & 1.20 & - & - & - & - & - & 0.23 \\
\hline Phenol & - & - & - & - & 0.06 & - & - & - & - & - & - & - & - & - \\
\hline Phytol & 0.34 & - & - & - & - & - & - & - & - & - & - & - & - & - \\
\hline$p$-vinyl-Guaiacol & 3.02 & - & - & - & - & - & - & - & - & - & - & - & - & - \\
\hline Selin-11-en- $4 \alpha$-ol & 0.16 & - & - & - & 1.16 & - & 0.13 & - & - & - & 0.09 & 0.20 & - & - \\
\hline trans-Carveol & 0.19 & 0.03 & - & - & - & - & - & - & - & - & - & 0.14 & 0.07 & - \\
\hline trans-p-Menth-2-en-1-ol & - & 0.01 & - & - & - & - & - & - & - & - & - & - & - & - \\
\hline$\alpha$-Acorenol & 0.16 & - & - & - & - & 0.06 & 0.81 & - & - & - & - & - & 0.19 & - \\
\hline$\alpha$-Bisabolol & 0.21 & 0.93 & - & - & - & 0.73 & 0.28 & 0.69 & - & 0.14 & 0.51 & 0.39 & 0.91 & 0.38 \\
\hline$\alpha$-Eudesmol & 0.21 & 1.05 & - & - & 0.96 & - & - & - & - & - & - & - & 0.39 & 0.06 \\
\hline$\alpha$-Muurolol & - & 0.09 & - & 0.62 & - & - & 1.83 & - & 1.72 & - & 0.13 & 0.24 & - & - \\
\hline$\beta$-Copaen- $4 \alpha$-ol & - & 1.25 & - & 0.96 & - & - & - & - & 0.29 & - & - & - & - & - \\
\hline$\gamma$-Eudesmol & - & 7.07 & - & - & - & - & - & - & 0.15 & - & - & - & - & - \\
\hline Sub Total & 25.0 & 24.4 & 5.92 & 34.4 & 31.3 & 44.9 & 26.5 & 6.29 & 34.8 & 2.08 & 30.4 & 11.8 & 11.5 & 7.90 \\
\hline \multicolumn{15}{|c|}{ Aldehyde and Ketone } \\
\hline$(2 E, 4 E)$-2,4-Decadienal & - & 0.07 & 0.42 & 0.25 & 0.06 & - & - & - & - & - & - & 0.05 & - & - \\
\hline (E)-2-Decenal & 0.85 & 0.04 & - & - & 0.06 & 0.18 & - & - & - & - & - & 0.32 & 0.14 & 0.07 \\
\hline (E)-2-Dodecenal & - & 0.72 & - & - & 0.58 & - & - & - & - & 0.20 & 0.09 & 0.24 & - & - \\
\hline (E)-2-Heptenal & - & - & - & - & 0.14 & - & 8.91 & 6.02 & - & 0.14 & - & - & - & - \\
\hline (E)-2-Undecenal & - & - & - & - & 0.09 & - & - & - & - & - & - & - & - & 0.24 \\
\hline (E)-4,5-Epoxy-2-decenal & - & - & - & - & - & - & - & - & - & - & - & 0.02 & - & - \\
\hline (E)- $\alpha$-Ionone & 0.28 & 1.43 & - & - & - & - & - & - & - & - & - & 0.05 & 0.05 & - \\
\hline$(E)$ - $\beta$-Ionone & 0.03 & 0.54 & - & - & 0.04 & - & - & - & - & - & - & - & - & - \\
\hline 1-Octen-3-one & - & - & - & - & - & - & - & - & - & - & - & - & 0.37 & - \\
\hline 2-Heptadecanone & - & 0.82 & - & - & - & 0.26 & - & 0.46 & 1.34 & - & 0.30 & 5.31 & 1.62 & 1.79 \\
\hline 2-Pentadecanone & 0.14 & 0.09 & - & - & - & 0.22 & - & - & 1.95 & 0.24 & - & 1.62 & - & - \\
\hline 2-Undecanone & - & - & - & - & - & - & - & - & 0.01 & - & - & 0.02 & 0.80 & - \\
\hline 6-Methyl-5-hepten-2-one & - & - & 5.40 & - & - & - & - & - & - & - & - & 3.11 & - & - \\
\hline Acetophenone & - & - & - & - & - & - & - & - & - & - & - & - & - & 0.65 \\
\hline Artemisia ketone & - & - & - & - & 0.79 & - & - & - & - & - & - & - & - & - \\
\hline Carvone & - & - & 0.34 & - & - & - & - & - & - & - & - & - & 0.14 & - \\
\hline cis-Cinnamaldehyde & - & - & - & - & 0.44 & - & - & - & - & - & - & - & - & - \\
\hline cis-Thujone & 0.26 & 0.12 & - & - & - & - & - & - & - & - & - & - & - & - \\
\hline Cumin aldehyde & 0.01 & - & - & - & - & - & - & - & - & - & - & - & - & - \\
\hline Farnesyl acetone, $(5 E, 9 E)$ & 3.09 & 0.88 & - & - & - & - & - & - & 0.23 & - & - & 0.28 & 0.49 & 0.06 \\
\hline Fenchone & 0.25 & - & - & - & - & - & - & - & - & - & - & - & - & - \\
\hline Geranial & - & 0.09 & - & - & 0.17 & - & 0.58 & 0.27 & - & - & - & - & - & - \\
\hline Hexadecanal & 1.06 & - & - & - & - & - & - & - & 0.69 & - & - & 0.17 & 1.72 & - \\
\hline Hexahydrofarnesylacetone & 0.07 & 0.95 & - & - & - & - & - & 0.15 & - & - & - & 1.55 & - & - \\
\hline Methional & - & - & - & - & 1.48 & - & - & - & - & - & - & - & - & - \\
\hline Nonanal & - & - & - & - & 1.84 & - & - & - & - & - & - & - & - & - \\
\hline Nootkatone & 0.24 & 1.03 & - & - & - & 0.34 & - & - & - & - & - & - & 0.13 & 0.15 \\
\hline Pentadecanal & - & 1.25 & - & - & - & 0.21 & 0.25 & 1.52 & - & - & 0.09 & 0.26 & - & 2.29 \\
\hline Pinocarvone & - & 0.52 & - & - & 2.69 & - & - & 0.38 & - & - & - & - & - & 0.96 \\
\hline Piperitenone & - & - & - & - & 2.58 & - & - & 1.09 & - & - & 0.43 & 8.52 & - & 0.13 \\
\hline Tetradecanal & 0.94 & 0.40 & 0.11 & - & - & - & 0.25 & 0.80 & 0.35 & - & 0.13 & 1.01 & 0.98 & 1.23 \\
\hline trans-Cinnamaldehyde & 0.13 & - & - & - & - & - & - & - & - & - & - & 0.03 & 0.14 & - \\
\hline trans-Dihydrocarvone & 0.02 & - & - & - & - & - & - & - & - & - & - & - & 0.04 & 0.05 \\
\hline Tridecanal & - & 0.25 & - & 2.20 & - & - & - & - & 1.27 & - & 0.94 & 0.93 & - & 0.27 \\
\hline Undecanal & - & - & 1.00 & 0.79 & - & - & - & - & 0.14 & - & - & 0.91 & - & - \\
\hline Valeranone & - & 0.35 & - & - & 1.52 & - & 1.74 & - & 0.48 & - & - & - & - & - \\
\hline Verbenone & - & - & - & 0.16 & - & - & - & - & - & - & - & - & - & - \\
\hline$\alpha$-Sinensal & - & - & - & - & - & - & - & - & - & - & - & 0.19 & - & - \\
\hline$\alpha$-Sinensal & - & 0.85 & 0.33 & 0.60 & - & 0.22 & - & - & - & - & - & 0.05 & 0.11 & - \\
\hline$\beta$-Oplopenone & 0.49 & 0.52 & - & - & - & - & - & - & - & - & - & - & 0.68 & 0.20 \\
\hline$\gamma$-Decalactone & 0.04 & - & - & - & - & 0.07 & - & 0.27 & - & 0.24 & 0.09 & - & 2.00 & - \\
\hline$\delta$-Decalactone & 0.07 & - & - & - & - & - & - & - & - & 1.29 & - & 2.03 & - & - \\
\hline Sub Total & 7.98 & 10.8 & 7.19 & 3.75 & 12.4 & 1.51 & 11.7 & 11.0 & 6.48 & 2.11 & 2.06 & 26.6 & 9.42 & 8.09 \\
\hline & & & & & rboxyli & acid & & & & & & & & \\
\hline 2-methyl-Butanoic acid & 0.03 & - & - & - & - & - & - & - & - & - & - & - & - & - \\
\hline (Z)-Hexadec-9-enoic acid & 0.42 & - & - & - & - & 2.28 & 0.13 & 3.27 & 0.06 & 0.24 & - & - & 0.82 & 1.00 \\
\hline Decanoic acid & 0.70 & - & - & - & - & - & - & - & - & - & 0.13 & 0.44 & - & - \\
\hline Heptanoic acid & 0.16 & - & - & - & 0.72 & - & - & - & - & - & - & - & - & - \\
\hline Hexadecanoic acid & 0.56 & - & 0.36 & 0.60 & - & - & 1.09 & - & 0.92 & 0.14 & - & 0.61 & 0.21 & 0.44 \\
\hline Hexanoic acid & - & 0.41 & - & - & - & - & - & - & - & - & - & 3.30 & 1.84 & 13.34 \\
\hline Linoleic acid & - & - & - & - & - & - & - & - & - & - & - & 0.11 & - & - \\
\hline Nonanoic acid & - & - & 0.27 & - & - & - & - & - & 0.10 & - & - & - & - & - \\
\hline Octadecanoic acid & 0.27 & 0.53 & - & - & - & - & - & - & 0.06 & 0.07 & - & - & - & 0.10 \\
\hline Octanoic acid & - & - & - & - & 0.10 & - & - & - & - & - & - & - & - & - \\
\hline Oleic acid & 0.35 & - & - & - & - & - & - & - & - & - & - & - & 0.47 & - \\
\hline Pentadecanoic acid & 0.10 & - & - & - & - & - & - & 0.43 & 0.21 & - & 0.47 & 0.39 & 0.15 & 0.68 \\
\hline Tetradecanoic acid & - & 0.14 & 0.49 & 0.74 & - & - & - & - & - & 0.68 & - & 0.09 & - & 0.05 \\
\hline Tridecanoic acid & 0.28 & 0.70 & 0.50 & - & - & 1.81 & - & - & - & - & - & 0.06 & 1.57 & 0.31 \\
\hline Undecanoic acid & - & 0.28 & - & - & 1.01 & - & - & 0.40 & - & - & - & - & - & - \\
\hline Sub Total & 2.88 & 2.05 & 1.62 & 1.34 & 1.83 & 4.09 & 1.22 & 4.11 & 1.35 & 1.12 & 0.60 & 5.01 & 5.07 & 15.9 \\
\hline
\end{tabular}


Continue from Table

\begin{tabular}{|c|c|c|c|c|c|c|c|c|c|c|c|c|c|c|}
\hline \multicolumn{15}{|c|}{ Esters } \\
\hline$(2 E, 6 E)$-Farnesyl acetate & - & 0.33 & - & - & - & - & - & 0.65 & 0.06 & - & 1.80 & - & 2.57 & 0.66 \\
\hline (Z)-3-Hexenyl benzoate & - & - & - & - & 1.24 & - & - & 0.40 & 0.13 & - & - & 0.34 & 1.87 & 0.55 \\
\hline 2-Methylpropyl 3-methylbutanoate & 2.42 & - & - & - & 0.11 & - & - & - & 0.02 & 0.24 & 0.13 & - & - & - \\
\hline 2-Phenylethyl acetate & - & - & - & - & 0.04 & - & - & - & - & - & - & 0.22 & - & - \\
\hline Benzyl benzoate & - & - & - & - & 0.55 & - & - & - & - & - & - & - & 12.48 & 0.44 \\
\hline Benzyl salicylate & - & - & - & - & - & 1.15 & - & - & - & - & - & - & - & - \\
\hline Bornyl acetate & 0.07 & - & - & - & 0.47 & - & - & - & - & - & - & - & - & - \\
\hline Bornyl formate & - & - & 0.21 & - & - & - & - & - & 0.02 & - & - & - & - & - \\
\hline$\beta$-Phenylethyl tiglate & - & - & - & 0.32 & - & 0.09 & - & 1.90 & 0.06 & - & - & 0.39 & 0.23 & 0.05 \\
\hline Carvacrol acetate & - & 0.24 & 1.09 & 1.24 & 0.66 & - & - & - & - & - & 0.09 & - & - & 0.05 \\
\hline cis-Carvyl acetate & 2.70 & - & - & - & 0.34 & - & - & - & - & - & - & 0.11 & - & - \\
\hline cis-Chrysanthenyl acetate & - & 0.04 & - & - & - & - & - & - & - & - & - & - & - & - \\
\hline cis-Sabinene hydrate & 0.16 & - & - & - & - & - & - & - & - & - & - & - & - & - \\
\hline cis-Sesquisabinene hydrate & 0.48 & 0.03 & 0.22 & 2.15 & - & - & - & - & 0.08 & - & - & 0.29 & 0.47 & 0.11 \\
\hline Citronellyl butanoate & - & - & - & - & - & - & - & - & 0.34 & - & - & 0.42 & - & 0.15 \\
\hline Decyl acetate & - & - & - & - & - & - & - & - & - & - & - & - & 0.12 & - \\
\hline endo-Fenchyl acetate & 0.15 & - & - & - & 0.20 & - & - & - & 0.01 & - & - & - & - & - \\
\hline Ethyl decanoate & - & 0.12 & 0.38 & 0.50 & - & - & - & 36.63 & - & - & - & 1.39 & - & - \\
\hline Ethyl dodecanoate & 5.14 & 0.02 & - & - & - & - & 0.21 & - & 0.43 & - & - & - & 0.15 & 0.73 \\
\hline Ethyl hexadecanoate & - & - & - & - & - & - & - & - & 2.28 & 0.24 & 0.21 & 0.65 & - & - \\
\hline Ethyl hexanoate & - & - & 4.37 & - & - & 0.15 & - & - & 3.75 & - & - & - & - & - \\
\hline Ethyl linoleate & - & - & 0.27 & - & - & - & - & - & - & - & - & - & 0.41 & - \\
\hline Ethyl pentanoate & 0.64 & 0.38 & - & 11.17 & 1.47 & 4.45 & 8.78 & 7.39 & 3.76 & 31.71 & 33.80 & - & 1.82 & 15.85 \\
\hline Ethyl tetradecanoate & 0.30 & 1.74 & - & - & 1.36 & 0.08 & 0.89 & 0.96 & - & - & - & - & 0.46 & 16.26 \\
\hline Eugenol acetate & 0.13 & 1.82 & - & 19.75 & - & - & - & - & 0.18 & - & 0.43 & 0.72 & - & 0.12 \\
\hline Geranyl acetate & - & 0.42 & 0.31 & 0.84 & 0.34 & 0.35 & - & - & - & - & - & - & - & 0.15 \\
\hline Geranyl butanoate & 0.22 & - & 0.21 & 0.19 & - & - & 1.61 & 1.66 & - & - & - & - & - & 0.71 \\
\hline Geranyl isovalerate & - & - & - & - & - & - & - & 0.10 & 3.93 & - & 1.20 & - & 0.42 & 0.20 \\
\hline Geranyl propanoate & 0.14 & - & 0.52 & - & - & - & - & - & - & - & - & - & 0.04 & - \\
\hline Geranyl tiglate & 0.01 & - & - & - & - & 0.81 & - & - & - & - & - & 0.60 & - & - \\
\hline Hexyl 2-methyl butanoate & - & - & - & - & - & - & - & - & - & - & - & - & 0.03 & - \\
\hline Hexyl benzoate & - & 0.19 & - & - & - & - & - & 0.29 & 0.01 & - & 1.03 & 0.86 & 1.12 & 0.57 \\
\hline Isopentyl isovalerate & - & - & - & - & - & - & - & - & - & - & - & - & - & 0.48 \\
\hline Lavandulyl acetate & 0.66 & - & - & - & - & - & - & - & - & - & - & - & - & - \\
\hline Linalool acetate & - & - & - & - & - & - & - & - & - & - & - & 0.28 & - & - \\
\hline Linalool propanoate & 0.16 & 0.08 & - & - & - & - & - & - & - & - & - & - & - & - \\
\hline Menthyl acetate & - & - & - & - & - & - & - & - & - & - & - & - & 0.07 & - \\
\hline Methyl 3-phenylpropionate & - & - & - & 0.25 & 0.08 & - & - & - & - & - & - & 0.02 & 0.64 & - \\
\hline Methyl decanoate & 0.10 & - & - & - & - & - & - & - & 0.04 & - & - & - & - & - \\
\hline Methyl geranate & - & - & - & - & 0.03 & - & - & - & - & - & - & 0.57 & - & 0.33 \\
\hline Methyl hexadecanoate & 0.25 & - & - & - & - & 1.09 & - & 0.66 & - & - & - & 0.05 & 0.47 & - \\
\hline Methyl hexanoate & - & - & 0.60 & 0.22 & - & - & - & - & 0.51 & - & 0.47 & - & - & - \\
\hline Methyl linoleate & - & 0.86 & 0.29 & 0.26 & 0.88 & 1.08 & - & - & 0.45 & 0.48 & - & - & 0.60 & - \\
\hline Methyl octadecanoate & - & - & - & - & - & - & - & 0.60 & - & - & - & - & - & 0.09 \\
\hline Methyl oleate & - & 0.52 & - & - & - & 0.63 & - & - & - & - & 0.17 & - & - & - \\
\hline Methyl tetradecanoate & - & - & 0.93 & 2.23 & - & - & - & - & - & - & 0.34 & 0.10 & 0.21 & 0.10 \\
\hline Myrtenyl acetate & 0.10 & 0.17 & 0.12 & - & 0.04 & - & - & - & - & - & - & 0.37 & - & - \\
\hline Neryl propanoate & 0.01 & 0.07 & - & - & - & - & - & - & - & - & 0.13 & 0.08 & - & - \\
\hline Nonyl acetate & - & 0.20 & - & 0.59 & 1.03 & - & - & 0.27 & - & - & - & 0.11 & - & 1.16 \\
\hline Octanol acetate & 2.62 & - & - & - & - & - & - & - & - & - & - & - & 0.69 & - \\
\hline Phenylethyl 3-methylbutanoate & - & - & 0.19 & - & 0.05 & 0.39 & - & - & - & - & - & 0.06 & - & - \\
\hline Terpinen-4-ol acetate & 0.08 & - & - & - & - & - & - & - & - & - & 0.13 & 0.06 & - & - \\
\hline trans-Pinene hydrate & - & - & - & - & 0.18 & - & - & - & - & - & - & - & - & - \\
\hline$\alpha$-Terpinyl acetate & - & 7.72 & - & 0.52 & - & - & - & 0.85 & - & - & - & - & - & - \\
\hline $\begin{array}{r}\text { Sub Total } \\
\end{array}$ & 16.6 & 14.6 & 9.70 & 40.2 & $\mathbf{7 . 8 3}$ & 10.3 & 11.5 & 51.3 & 15.9 & 32.7 & 38.1 & 7.35 & 20.4 & 37.6 \\
\hline
\end{tabular}

1,8-Cineole

cis-Limonene oxide

cis-Linalool oxide, (furanoid)

Dehydro-1,8-cineole

Dendrolasin

Dill apiole

Dimethyl trisulfide

Humulene epoxide II

Indole

Manoyl oxide

Myristicin

Piperitenone oxide

trans-Limonene oxide

trans-Linalool oxide, (furanoid)

Miscellaneous Compound

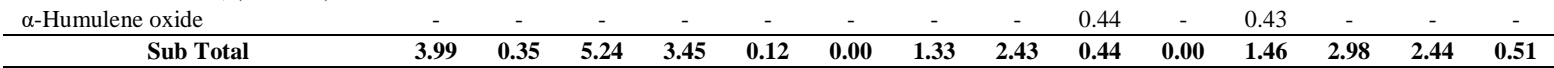

\begin{tabular}{|c|c|c|c|c|c|c|c|c|c|c|c|c|c|c|}
\hline \multicolumn{15}{|c|}{ Terpenes } \\
\hline (E)- $\beta$-Farnesene & - & - & - & 0.23 & - & - & - & - & - & - & - & - & - & - \\
\hline$(E, E)$ - $\alpha$-Farnesene & - & - & 0.86 & 1.24 & - & - & - & 0.40 & - & - & - & - & - & - \\
\hline
\end{tabular}




\begin{tabular}{|c|c|c|c|c|c|c|c|c|c|c|c|c|c|c|}
\hline \multicolumn{15}{|l|}{ Continue from Table 2} \\
\hline (Z)-Caryophyllene & 7.69 & - & - & - & - & - & - & - & - & - & - & - & - & - \\
\hline (Z)- $\gamma$-Bisabolene & - & - & - & - & - & - & 0.24 & - & - & - & - & - & - & - \\
\hline 2,5-Dimethoxy-p-cymene & - & - & - & - & - & - & - & 0.12 & - & - & - & - & - & - \\
\hline Abietatriene & 0.09 & 0.49 & - & - & - & - & - & - & 0.29 & 0.14 & - & - & - & - \\
\hline Alloaromadendrene & 0.19 & 0.45 & - & - & - & - & 1.15 & - & - & - & - & - & 0.05 & - \\
\hline$\alpha$-Pinene & - & - & 0.61 & - & 0.45 & - & - & - & 0.04 & - & - & 0.84 & 0.83 & 6.76 \\
\hline \multicolumn{15}{|l|}{ Continue.. } \\
\hline Aromadendrene & 0.11 & - & - & - & - & - & - & - & - & - & - & 0.14 & - & - \\
\hline$\beta$-Copaene & - & - & - & - & - & - & 0.35 & - & - & - & - & - & 0.09 & - \\
\hline$\beta$-Humulene & - & - & 0.22 & - & 0.56 & - & - & 0.12 & - & - & - & - & - & - \\
\hline Bicyclogermacrene & - & - & - & - & - & - & - & - & - & - & - & - & - & - \\
\hline Cadalene & 0.21 & - & - & - & 1.47 & - & - & 0.15 & - & - & - & - & - & - \\
\hline Chamazulene & 0.33 & 0.21 & - & - & 0.57 & 0.29 & - & - & - & 0.17 & - & 1.86 & - & - \\
\hline cis-Cadina-1(2),4-diene & - & 1.58 & - & - & 0.65 & - & - & 0.11 & 0.10 & - & - & - & - & - \\
\hline cis-Muurola-4 (14),5-diene & - & - & - & - & 2.59 & - & - & - & - & - & - & - & - & - \\
\hline cis- $\alpha$-Bergamotene & - & - & - & - & - & - & - & - & 0.75 & 0.14 & - & 0.54 & - & - \\
\hline cis- $\beta$-Farnesene & - & - & - & - & 0.33 & 0.04 & - & 0.09 & - & 0.10 & 0.09 & 0.05 & 0.43 & - \\
\hline Cuparene & - & - & - & - & - & - & - & - & - & - & - & - & - & - \\
\hline$\delta$-Elemene & - & - & - & - & - & - & - & - & - & - & - & - & - & - \\
\hline Elemicin & 0.52 & - & - & - & - & - & - & - & - & - & - & - & - & - \\
\hline Germacrene B & - & - & - & - & - & 0.93 & - & - & - & - & - & - & - & - \\
\hline Isoitalicene & - & 1.01 & - & - & 0.13 & - & - & - & - & - & - & - & - & - \\
\hline$m$-Cymene & - & - & - & - & - & - & - & - & - & - & - & - & - & 0.22 \\
\hline Myrcene & - & - & - & 2.79 & - & - & - & - & - & - & 3.35 & - & - & - \\
\hline$o$-Cymene & 0.11 & - & - & - & - & - & - & - & - & - & - & - & - & - \\
\hline Santolina triene & 0.83 & - & - & - & - & - & - & - & - & - & - & - & - & - \\
\hline Thuja-2,4(10)-diene & 0.19 & 0.66 & - & - & 0.94 & - & 8.40 & - & 0.02 & 0.10 & - & - & 1.12 & - \\
\hline trans- $\alpha$-Bergamotene & - & 0.08 & - & - & 1.47 & - & - & - & - & - & - & - & - & - \\
\hline Tricyclene & - & - & - & - & - & - & - & - & - & - & - & - & - & - \\
\hline Verbenene & - & - & - & - & - & - & - & 3.61 & - & - & - & - & - & - \\
\hline$\alpha$-Amorphene & 0.09 & 0.03 & - & - & - & - & - & - & - & - & - & - & - & - \\
\hline$\alpha$-Cadinene & - & 0.68 & 49.39 & 4.35 & - & - & - & - & - & - & - & - & 0.93 & 0.12 \\
\hline$\alpha$-Cedrene & 5.10 & 0.02 & 5.20 & - & - & - & - & - & - & - & 0.30 & - & 0.09 & 0.06 \\
\hline$\alpha$-Cubebene & - & 3.01 & - & - & - & - & - & - & - & - & - & 0.02 & - & - \\
\hline$\alpha$-Fenchene & 0.13 & - & - & - & - & 3.41 & - & - & - & 0.37 & 0.17 & - & - & - \\
\hline$\alpha$-Guaiene & - & - & - & - & - & - & - & - & - & - & - & - & - & - \\
\hline$\alpha$-Gurjunene & - & - & - & - & - & - & - & - & - & - & - & 0.05 & - & - \\
\hline$\alpha$-Himachalene & 0.15 & 0.17 & - & - & - & - & 0.24 & 0.11 & - & - & - & - & - & - \\
\hline$\alpha$-Humulene & 0.24 & 0.18 & - & - & - & - & - & - & - & - & - & 0.33 & - & - \\
\hline$\alpha$-Thujene & 0.51 & - & 1.00 & 3.25 & - & 1.83 & 3.91 & 3.16 & 1.17 & 16.74 & 14.55 & 0.35 & - & - \\
\hline$\alpha$-Ylangene & 0.03 & - & - & - & - & - & - & - & 0.22 & - & - & 0.18 & - & - \\
\hline$\alpha$-Zingiberene & - & 0.18 & - & - & - & - & - & 0.20 & - & - & 0.56 & - & 0.08 & - \\
\hline$\beta$-Acoradiene & 0.02 & 0.09 & - & - & 1.03 & - & - & - & - & - & - & - & 0.07 & - \\
\hline$\beta$-Bisabolene & - & - & - & - & - & - & - & - & 0.25 & - & - & - & 2.30 & 0.19 \\
\hline$\beta$-Calacorene & - & 0.19 & - & - & - & - & - & - & 0.06 & 0.07 & 1.16 & 3.43 & 0.15 & - \\
\hline$\beta$-Cedrene & 0.08 & - & 8.18 & - & 2.56 & - & - & - & - & - & - & - & - & - \\
\hline$\beta$-Chamigrene & - & - & - & - & - & - & - & - & - & - & - & - & - & - \\
\hline$\beta$-Cubebene & 2.30 & - & - & - & 0.09 & - & - & - & 0.07 & - & 0.09 & - & - & - \\
\hline$\beta$-Elemene & - & 1.16 & - & - & 0.11 & - & - & 0.31 & 0.09 & - & 0.19 & 2.94 & - & 0.22 \\
\hline$\beta$-Gurjunene & 0.04 & 0.33 & - & - & 0.29 & - & - & - & - & - & - & 0.07 & - & - \\
\hline$\beta$-Himachalene & 0.99 & 0.10 & - & - & - & - & - & - & - & - & - & 0.60 & - & - \\
\hline$\beta$-Phellandrene & 9.56 & - & - & - & - & - & - & - & - & - & - & - & - & - \\
\hline$\beta$-Santalene & 0.05 & - & - & - & - & - & - & - & - & - & - & 0.11 & - & - \\
\hline$\gamma$-Curcumene & 0.08 & 0.04 & - & - & - & - & - & - & - & - & - & 0.06 & - & - \\
\hline$\gamma$-Elemene & 0.61 & - & - & - & - & - & - & - & - & - & - & - & - & - \\
\hline Sub Total & 31.3 & 10.7 & 65.5 & 11.9 & 13.2 & 6.50 & 14.3 & 8.68 & 3.07 & 18.1 & 20.5 & 11.6 & 7.66 & 7.57 \\
\hline Total & 87.7 & 62.9 & 95.1 & 95.1 & 66.9 & 67.2 & 66.5 & 83.8 & 62.0 & 56.0 & 93.1 & 65.3 & 56.5 & 77.5 \\
\hline
\end{tabular}

Notes : PM - M. acuminata colla 'sucrier', PN - M. acuminata $\times$ balbisiana colla 'saba', PT - M. acuminata $\times$ balbisiana 'horn plantain', PE - M. acuminata colla 'gros michel', PO - M. acuminata colla 'inarnibal', PJ - M. acuminata colla 'red', PB - M. acuminata colla 'lakatan', S-Peels, F-Flesh

Three compounds in the alcohol group namely, 1tetradecanol, $(2 E, 6 E)$-farnesol and epi-1-cubenol are the major components that were found in essential oil almost all seven species of Musa's essential oils. 1Tetradecanol is the major component found in M. acuminata $\times$ balbisiana colla 'saba' peels (5.22\%) and M. acuminata colla 'inarnibal' peels (2.54\%). Alcohol group contributes to the greenwoody smell in the Musa spp. (Aurore et al., 2011).

Phenylpropanoids compounds such as eugenol, methyl eugenol and carveol were also found in the essential oil for almost all seven Musa spp. However, carveol is identified in the Musa spp. in Malaysia but was not detected in M. acuminata colla 'gros michel' in Madeira Island (Nogueira et al., 2003). The highest percentage of eugenol (8.83\%) was found in $M$. acuminata colla 'sucrier' flesh oil.

Ester group is an important volatile component in Musa spp. as this group plays a major role in the aroma of fully ripe banana fruits and responsible for the essence that carry by Musa spp. (Selli et al., 2012). A total of 54 components were identified in the 
essential oils are from the ester group, where acetate esters were found in a higher concentration compared to the other esters. Carvacrol acetate, methyl hexanoate, methyl-3-phenylpropionate, eugenol acetate and ethyl pentanoate were the components from ester group that were identified in all of the studied species. However, the percentage abundance of the components differs between each species.

Ethyl pentanoate present in all Musa spp.'s fruit with the highest percentage of the component is identified in M. acuminata colla 'gros michel' flesh (33.8\%). Besides that, eugenol acetate is also a common ester that present in the essential oil of Musa spp.'s fruit. This component was detected in Musa spp. essential oils with the highest abundance in $M$. acuminata $\times$ balbisiana colla 'saba' (19.75\%). However, the component was not detected in the essential oil of $M$. acuminata $\times$ balbisiana 'horn plantain' and $M$. acuminata colla 'inarnibal'.

Besides alcohol, carbonyl components are also contributed to the aroma characteristic in Musa spp. In this study, a total of 42 components of carbonyl group were identified in Musa spp.'s fruits essential oil. Hexadecanal and (E)-2-hexanal are minor constituents detected in $M$. acuminata colla 'inarnibal' flesh $(0.14 \%)$ and $M$. acuminata colla 'gros michel' peels $(0.17 \%)$. In contrast, previous studies found that the carbonyl group was the major constituent in their research (Shiota, 1993).

Tridecanoic acid and hexadecanoic acid are carboxylic acids that were found in all Musa spp.'s fruit essential oils. Hexadecanoic acid were also found in the study done by Nogueira et al., (2003), while tridecanoic acid is not reported in any previous studies. These carboxylic acids are associated with the aroma of the banana and the ripening of banana (Pino \& Febles, 2013).

The chemical constituents in the essential oils of Musa spp. are different due to their different maturity stage of the fruits (Brat et al., 2004; Macku and Jennings 1987). Pentan-2-one was reported as the major constituent in Musa spp. from Philippines (Shiota, 1993). However, in this study the compounds were not detected in all seven Musa spp. that were studied. As shown in Table 1, the major components percentage abundance of seven Musa spp.'s fruit is in a range of 5.26\% to $49.39 \%$. Most of the major components present in the essential oils studied are from the ester and alcohol groups.
Besides that, the differences between chemical constituents and the percentage of the constituent in all seven Musa spp. studied are different from the literature. This may be due to the different extraction method used in both studies. In this study, hydrodistillation method was employed for the extraction of the essential oils from Musa spp. This method requires the oil collector to be heated and this may have resulted in the degradation of volatile and thermolabile compounds by the thermal reaction which include transformation reaction and oxidation reactions (Chen et al., 2014). This explains why in previous studies researchers prefer headspace-solid phase microextraction method in isolating volatile oil of Musa spp.

\section{Cluster Analyses}

The statistical analysis of the essential oils was carried out to show the relationship between the studied species. The analysis was carried out by SPSS version 22 on the flesh and peels the essential oils. The dendrogram for the flesh and peels are shown in Figure 1 and Figure 2, respectively. Figure 1 shows that there is a strong relationship between the flesh oil of $M$. acuminata colla 'inarnibal', $M$. acuminata $\times$ balbisiana 'horn plantain', M. acuminata colla 'lakatan', M. acuminata colla 'red' and $M$. acuminata colla 'sucrier'.

M. acuminata colla 'gros michel' and Musa acuminata colla 'red' also showed significant relationship between the two species. In contrast, $M$. acuminata $\times$ balbisiana colla 'saba' have no correlation with the other six species. The species that were studied had a uniform qualitative essential oil composition but differed in quantitative content. This difference in morphology is also one of the factors that causes the difference in the chemical variability among the species (Joshi et al., 2009).

Based on Figure 2, it shows that there is a strong relationship between $M$. acuminata colla 'sucrier', M. acuminata colla 'gros michel', M. acuminata colla 'inarnibal' and M. acuminata colla 'lakatan'. $M$. acuminata $\times$ balbisiana colla 'saba' and $M$. acuminata $\times$ balbisiana 'horn plantain' also showed a significant relationship between the cultivar. However, for $M$. acuminata colla 'red' it has no colleration with the other six species. A strong relationship between M. acuminata colla 'sucrier', M. acuminata colla 'gros michel', M. acuminata colla 'inarnibal' and M. acuminata colla 'lakatan' was due to the same AA genome group, while for $M$. acuminata $\times$ balbisiana colla 'saba' and $M$. 


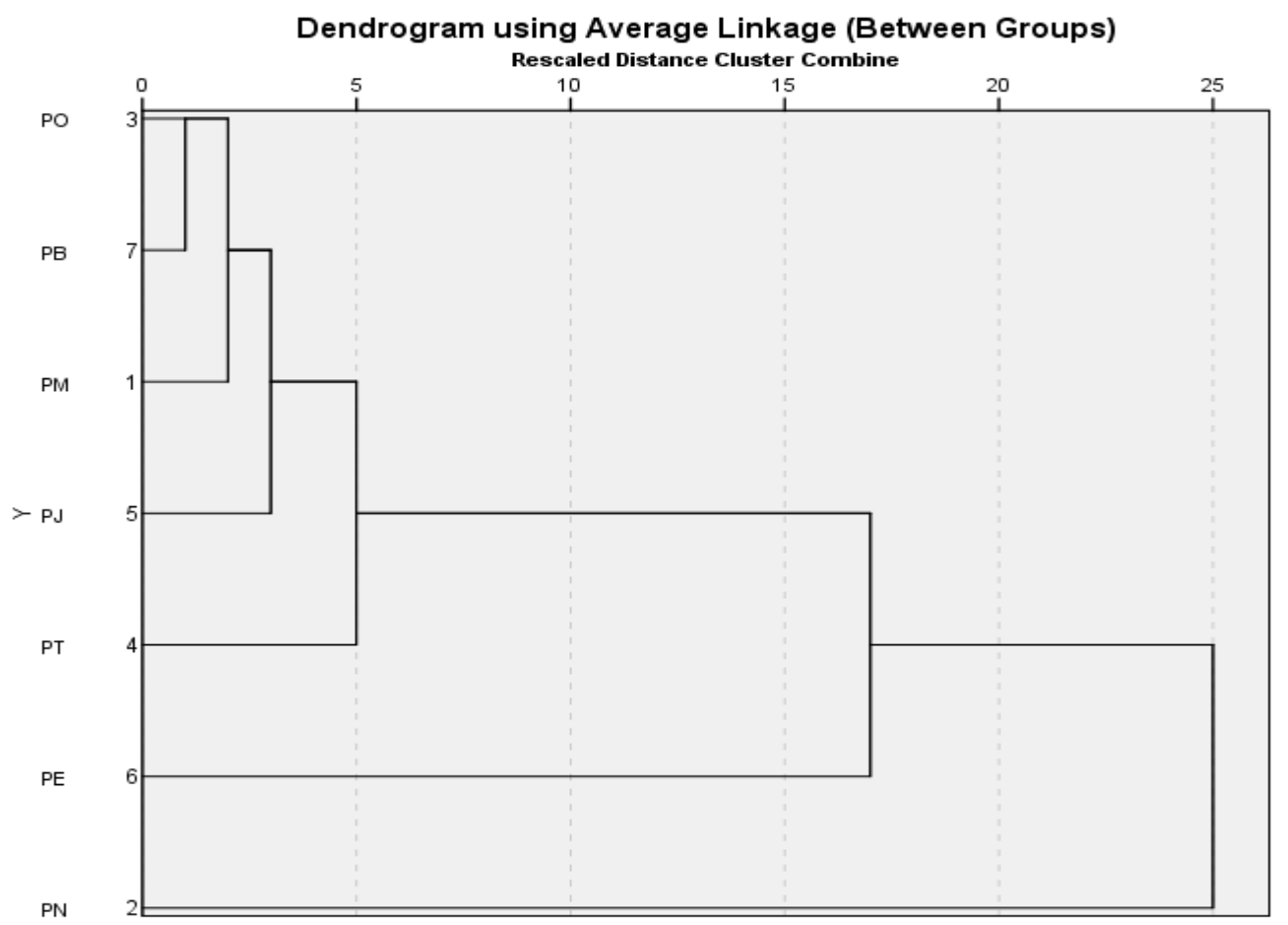

Figure 1. Dendogram of cluster analysis for the flesh oils of seven Musa spp.'s fruits

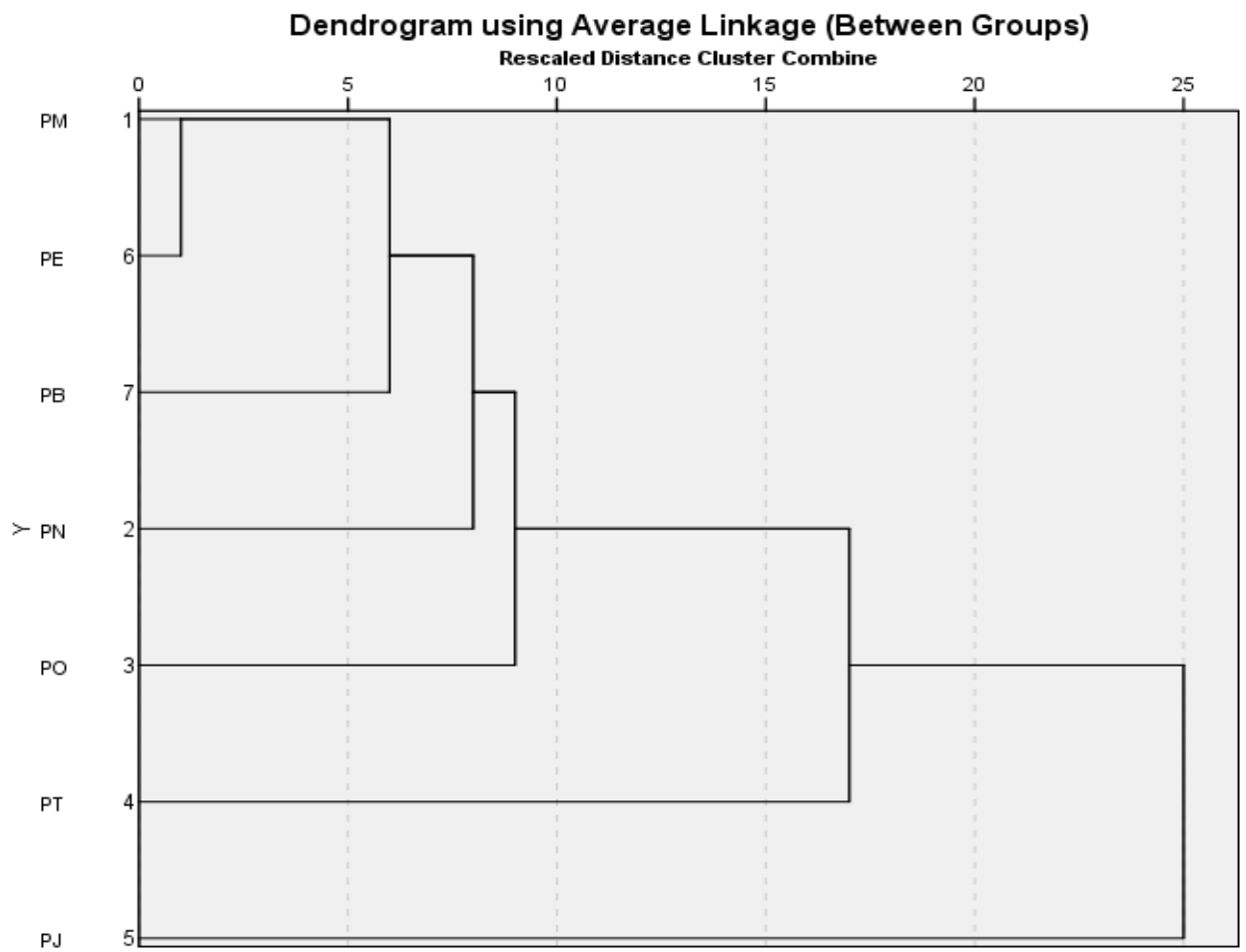

Figure 2. Dendogram of cluster analysis for the peels oils of seven Musa spp.'s fruits 
acuminata $\times$ balbisiana 'horn plantain' are from the combination of $\mathrm{AA}$ and $\mathrm{BB}$ genome group. $\mathrm{BB}$ genome group has different unique characteristic and varies with each other (Ploetz et al., 2007).

\section{Brine Shrimp Toxicity}

Toxicity against Artemia salina had been carried out on the essential oils of M. acuminata colla 'sucrier' (flesh and peels), M. acuminata $\times$ balbisiana colla 'saba' (flesh and peels), M. acuminata $\times$ balbisiana 'horn plantain' (flesh and peels) and $M$. acuminata colla 'inarnibal' (peels). After 24 hours, the number of death Artemia salina was observed and recorded. The percentage of death for Artemia salina against the essential oil of Musa spp. at different concentrations are shown in
Figure 3.

According to Kalala et al. (2015), LC $\mathrm{C}_{50}$ value greater than $100 \mathrm{ppm}$ is considered nontoxic, while $\mathrm{LC}_{50}$ below or equal to $99 \mathrm{ppm}$ is considered toxic. All seven sample tested were interpreted as non-toxic ( $\mathrm{LC}_{50}>100 \mathrm{ppm}$ ). Based on the results, the samples showed a dose of dependent toxicity activity indicating the potential of the sample to be studied for their antitumor properties. McLaughlin (1991) stated the toxicity of the sample on brine shrimp test was correlated with the antitumor activity. Besides, another study by Bagya et al. (2011) also showed a good correlation between toxicity and brine shrimp with antitumor properties of Kaempferia galangal ethanolic extract.

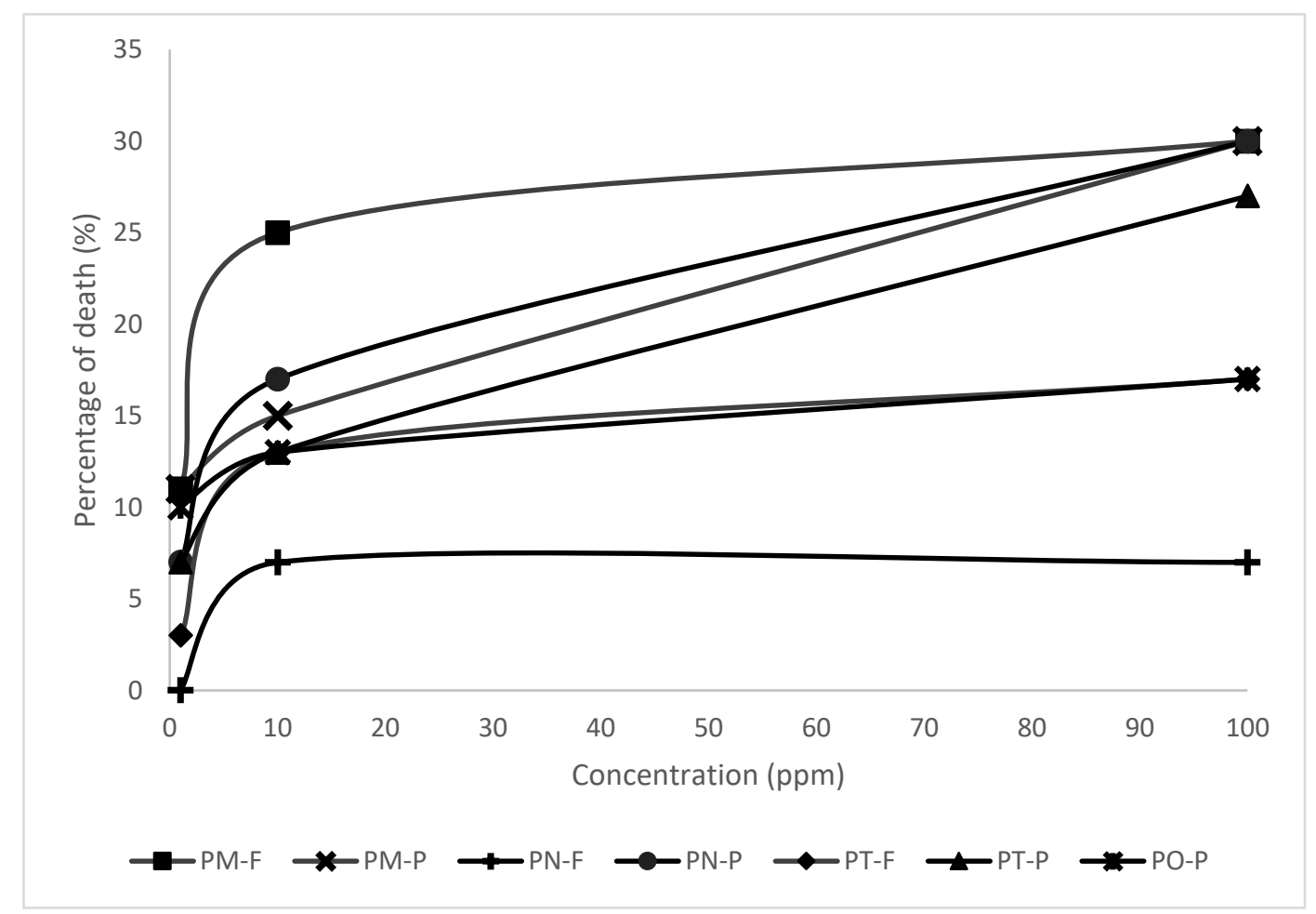

Figure 3. Percentage of death brine shrimp against concentration graph

Notes : PM-F - Musa acuminata colla 'sucrier' flesh oil, PM-P - Musa acuminata colla 'sucrier' peels oil, PNF - Musa acuminata $\times$ balbisiana colla 'saba' flesh oil, PN-P - Musa acuminata $\times$ balbisiana colla 'saba' peels oil, PT-F - Musa acuminata $\times$ balbisiana 'horn plantain' flesh oil, PT-S - Musa acuminata $\times$ balbisiana 'horn plantain' peels oil, PO-P - Musa acuminata colla 'inarnibal' peels oil

\section{Antibacterial Assay}

An antibacterial test had been carried out on essential oils of M. acuminata colla 'sucrier' (peels and flesh), M. acuminata $\times$ balbisiana 'horn plantain' (peels and flesh), M. acuminata colla 'gros michel' (peels and flesh) and $M$. 
acuminata $\times$ balbisiana colla 'saba' (peels and flesh). Three types of bacteria used were Staphylococcus aureus, Staphylococcus epidermidis and Pseudomonas aeruginosa. Sample with a concentration of $100 \mu \mathrm{g} \mathrm{disc}{ }^{-1}$ was used for the antibacterial activity. However, there was no inhibition zone observed for all the essential oils tested. This may due to the concentration of the tested essential oils $\left(100 \mu \mathrm{g} \quad \operatorname{disc}^{-1}\right)$ that was insufficient for antibacterial activity.

Research done by Fagbemi et al. (2009) on unripe ethanol extract of $M$. sapientum showed minimum inhibitory concentration of $32000 \mathrm{ug} / \mathrm{ml}$ on the bacteria Escherichia coli and $S$. aureus. The difference between the method of extracting essential oils and hydrodistillation and solvent extraction can influence the antimicrobial properties. This was shown by the essential oils extracted by hexane that has greater antimicrobial activity compared to steam hydrodistillation of the corresponding essential oil (Packiyasothy \& Kyle, 2002).

\section{CONCLUSION}

In conclusion, the study revealed that ester and alcohol groups are the most dominant components in the flesh and peel oils of Musa spp. This has contributed to the biological activities of the essential oils. Weak brine shrimp toxicity of the tested samples may hint the potential of the essential oil as an antitumor medicine. Further studies on essential oil of Musa spp. should focus on their antitumor properties.

\section{ACKNOWLEDGMENTS}

The authors thank the Faculty of Resource Science and Technology for the research facilities provided. The authors thank Mr. Sekudan Tedong for identification samples.

\section{REFERENCES}

Aurore, G., Ginies, C., Ganou-parfait, B., Renard, C. M. \& Fahrasmane, L. (2011). Comparative study of free and glycoconjugated volatile compounds of three banana cultivars from French West indies: Cavendish, Frayssinette and Plantain. Food chemistry, 129(1): 28-34.
Aazza, S., Lyoussi, B., Megìas, C., Cortès-Giraldo, I., Vioque, J. \& Figueiredo, A.C. (2014). Antioxidant, anti-inflammatory and antiproliferative activities of Moroccan commercial essential oils. Natural Product Communications, 9(4): 587-94.

Bagya S.K., Rajashree, P.V. \& Sam, K.G. (2011). Preliminary anticancer screening and standarization of some indigenous medicinal plants using cell-biology and molecular biotechnology based models. Research Journal of Medicinal Plant, 5(6): 728-737.

Brat, P., Yahia, A., Chillet, M., Bugaud, C., Bakry, F., Reynes, M., \& Brillouet, J.M. (2004). Influence of cultivar, growth altitude and maturity stage on banana volatile compound composition. Fruits, 59(2): 75-82.

Buchbauer, G. (2000). The detailed analysis of essential oils leads to understanding of their properties. Perfumer and Flavorist, 64-76.

Calcabrini, A., Stringaro, A., Toccacieli, L., Meschini, S., Marra, M. \& Colone M. (2004). Terpinen-4-ol, the main component of Melaleuca alternifolia (tea tree) oil inhibits the in vitro growth of human melanoma cells. Journal of Investigative Dermatology, 122: 349-60.

Chen, Y., Wu, J., Xu, Y., Fu, M. \& Xiao, G. (2014). Effect of second cooling on the chemical components of essential oils from orange peel (Citrus sinensis). Journal of Agricultural and Food Chemistry, 62(35): 8786-8790.

Di, C.E., Di Bartolomeo, S., Delli Pizzi, P., Di Giulio, M., Grande, R., Nostro, A. \& Cellini, L. (2012). Activity of tea tree oil and Nerolidol alone or in combination against Pediculus capitis (Head Lice) and its eggs. Parasitol Research, 111(5): 1985-92.

Dutra, T.V., Castro, J.C., Menezes, J.L., Ramos, T.R., do Prado, I.D., Junior, M.M., Mikcha, J.M.G. \& Filho, B.A.A. (2019). Bioactivity of oregano (Origanum vulgare) essential oil against Alicyclobacillus spp. Industrial Crops and Products, 129: 345-349.

Fagbemi, J.F., Ugoji, E., Adenipekun, T. \& Adelowotan, O. (2009). Evaluation of the antimicrobial properties of unripe banana (Musa sapientum L.), lemon grass (Cymbopogon citratus S.) and turmeric (Curcuma longa L.) on pathogens. African Journal of Biotechnology; 8(7): 1176-1182. 
Gkinis, G., Tzakou, O., Iliopoulou, D. \& Roussis, V. (2003). Chemical composition and biological activity of Nepeta parnassica oils and isolated Nepetalactones. Zeitschrift für Naturforschung $C$, 58: 681-686.

Guylène, A., Berthe, P. \& Louis, F. (2008). Bananas, raw materials for making processed food product. Trends in Food Science and Technology, 20: 78-91.

Jordán, M.J., Shaw, P.E. \& Goodner, K.L. (2001). Volatile components in aqueous essence and fresh fruit of cucumis melo cv. Athena (muskmelon) by GC-MS and GC-O. Journal of Agricultural and Food Chemistry, 49(12): 5929-5933.

Joshi, S.C., Padalia, R.C., Bisht, D.S. \& Mathela, C.S. (2009). Terpenoid diversity in the leaf essential oils of Himalayan Lauraceae species. Chemistry and Biodiversity, 6(9): 1362-1373.

Kalala, W., Mwakigonja, A., Maregesi, S., Msengwa, Z. \& Mahunnah, R. (2015). Brine shrimp lethality and acute oral toxicity of Commiphora swynertonii (burrt) exudate. Pyrex Journal of Medicinal Plant Research, 1(3): 10-18.

Koh, K.J., Pearce, A.L., Marshman, G., FinlayJones, J.J. \& Hart, P.H. (2002). Tea tree oil reduces histamine induced skin inflammation. British Journal of Dermatology, 147: 1212-1217.

Macku, C. \& Jennings, W.G. (1987). Production of volatiles by ripening bananas. Journal of Agricultural and Food Chemistry, 35: 845-848.

McLaughlin, J.L., 1991. Crown-gall Tumors in Potato Discs and Brine Shrimp Lethality: Two Simple Bioassays for Higher Plant Screening and Fractionation. In: Hostettmann, K. (Ed.), Methods in Plant Biochemistry, London: Academic Press.

McLaughlin, J.L., Rogers L.L. \& Anderson, J.E. (1998). The use of biological assays to evaluate botanicals. Drug Information Journal, 32: 513524.

Murbach Teles Andrade, B.F., Conti, B.J., Santiago, K.B., Fernandes J.A. \& Sforcin, J.M. (2014). Cymbopogon martinii essential oil and geraniol at noncytotoxic concentrations exerted immunomodulatory anti-inflammatory effects in human monocytes. Journal of Pharmaceutics and Pharmacology, 66(10): 1491-1496.
Nogueira, J.M.F., Fernandes, P.J.P. \& Nascimento, A.M.D. (2003). Composition of volatiles of banana cultivars from Madeira Island. Phytochemical Analysis, 14: 87-90.

O'Hara, M., Kiefer, D., Farrell, K. \& Kemper, K. (1998). A review of 12 commonly used medicinal plant. Archives of Family Medicine, 523-536.

Orhan, I. (2001). Biological activities of Musa species. Journal of the Faculty of Pharmacy, 3950.

Pari, L. \& Umamaheswari, J. (2000). Antihyperglycaemic activity of Musa sapientum flowers: Effect on lipid peroxidation in Alloxan Diabetic rats. Phytotherapy Research, 136-138.

Packiyasothy, E.V.\& Kyle, S. (2002). Antimicrobial properties of some herb essential oils. Food Australia; 54(9): 384-387.

Pérez, A.G., Cert, A., Rios, J.J. \& Olias, J.M. (1997). Free and glycosidically bound volatile compounds from two bananas cultivars: Valery and Pequenã Enna. Journal of Agricultural and Food Chemistry, 45: 4393-4397.

Pino, J.A. \& Febles, Y. (2013). Odour-active compounds in banana fruit cv. giant cavendish. Food Chemistry, 141(2): 795-801.

Ploetz, R.C., Kepler, A.K., Daniells, J. \& Nelson, S.C., (2007). Banana and plantain- an overview on Pacific island cultivars. Agroforestry, 1-27.

Selli, S., Gubbuk, H., Kafkas, E. \& Gunes, E. (2012). Comparison of aroma compounds in dwarf cavendish banana (Musa spp. AAA) grown from open-field and protected cultivation area. Scientia Horticulture, 141: 76-82.

Shiota, H. (1993). New esteric components in the volatiles of banana fruit (Musa sapientum L.). Journal of Agricultural and Food Chemistry, 41(11): 2056-2062.

Sienkiewicz, M., Glowacka, A., Poznanskakurowska, K., Kaszuba, A., urbaniak, A. \& Kowalczyk, E. (2015). The effect of clary sage oil on Staphylococci responsible for wound infection. Advances in Dermatology and Allergology, 32(1): 21-26.

Sim, S.F., Sinang, F.M. Diana, K., Felecia, C. Melissa, E.D. Vanessa, R.N.L. \& Noor, S.A.M. (2013). Combining essential oil of Piper betle and Myristica fragrans for enhanced antimicrobial properties. Borneo Journal of Resource Science and Technology, 3(1): 35-42. 